\title{
Resonant Absorption in GaAs-Based Nanowires by Means of Photo-Acoustic Spectroscopy
}

\author{
E. Petronijevic ${ }^{1}$ (D) G. Leahu ${ }^{1} \cdot$ A. Belardini $^{1}$. \\ M. Centini ${ }^{1}$ - R. Li Voti ${ }^{1}$ - T. Hakkarainen ${ }^{2}$. \\ E. Koivusalo $^{2}$ - M. Guina ${ }^{2}$. C. Sibilia ${ }^{1}$
}

Received: 3 November 2017 / Accepted: 12 February 2018

(C) Springer Science+Business Media, LLC, part of Springer Nature 2018

\begin{abstract}
Semiconductor nanowires made of high refractive index materials can couple the incoming light to specific waveguide modes that offer resonant absorption enhancement under the bandgap wavelength, essential for light harvesting, lasing and detection applications. Moreover, the non-trivial ellipticity of such modes can offer near field interactions with chiral molecules, governed by near chiral field. These modes are therefore very important to detect. Here, we present the photo-acoustic spectroscopy as a low-cost, reliable, sensitive and scattering-free tool to measure the spectral position and absorption efficiency of these modes. The investigated samples are hexagonal nanowires with GaAs core; the fabrication by means of lithographyfree molecular beam epitaxy provides controllable and uniform dimensions that allow for the excitation of the fundamental resonant mode around $800 \mathrm{~nm}$. We show that the modulation frequency increase leads to the discrimination of the resonant mode absorption from the overall absorption of the substrate. As the experimental data are in great agreement with numerical simulations, the design can be optimized and followed by photo-acoustic characterization for a specific application.
\end{abstract}

Keywords GaAs · Nanowires · Photo-acoustic technique

This article is part of the selected papers presented at the 19th International Conference on Photoacoustic and Photothermal Phenomena.

$\bowtie \quad$ E. Petronijevic

emilija.petronijevic@uniroma1.it

1 Department S.B.A.I, Sapienza Università di Roma, Via A. Scarpa 14, 00161 Rome, Italy

2 Optoelectronics Research Centre, Tampere University of Technology, Korkeakoulunkatu 3, 33720 Tampere, Finland 


\section{Introduction}

Semiconductor nanowires (NWs) with high refractive index and high aspect ratio can couple the incoming light to the set of waveguide modes, similar to the ones defined in infinite cylindrical dielectric waveguides [1]. Under the bandgap wavelength, these modes experience loss and lead to the resonant absorption, leading to many fields where they can be applied: photovoltaics [2-5], light sources [6, 7], photodetectors [8], waveguides [9] etc. Especially important are the reports on NW lasers working at room temperature [10], their integration on Si [11] and highly efficient NW solar cells [12]. Numerically, the NW waveguide modes have been vastly investigated [13-17]. The properties of the confined fields have also recently lead to some new prospects in spin angular momentum formation [18], and near chiral field manipulation [19, 20]. Finally, semiconductor NW hybridization with metallic layers has recently shown effects such as circular dichroism [21].

The conventional characterization of the NW absorption usually relies on the measurements of transmission/reflection [22], photoluminescence [23-25] or photocurrents [26]. However, these techniques require post-processing as they do not directly provide the absorption efficiency. Moreover, the results can be affected by the vast scattering in the disordered samples. Recently, the photo-acoustic spectroscopy was applied to measure the absorption edge in disordered GaAsBi/GaAs NWs [27]. In [17] we showed, for the first time to our knowledge, that highly uniform NW samples can couple light to the discrete absorption modes in the visible and that their spectral position and efficiency can be reliably characterized by this technique. Here, we discuss the influence of the substrate on the absorption in photo-acoustic measurements, and the numerical set-up which gives great agreement between the theory and the experiment.

\section{Nanowire Waveguide Modes and Resonant Absorption}

Firstly, we present the numerical approach for the investigation of the resonant absorption modes of GaAs-based NWs. We define the geometry following the real samples' properties from [28] that will then be photo-acoustically characterized. The samples were fabricated by means of a recently developed, lithography-free molecular beam epitaxy (MBE) that provides high quality and uniformity GaAs NW ensembles on p-Si(111) wafers. As there is no periodical pattering of the NW position, the overall optical response of the sample is predominantly due to the interaction of a single NW with light; here, we investigate this interaction in the visible part of the spectrum.

The simulated structure is a single GaAs-based NW with hexagonal cross section, sketched in Fig. 1a. It consists of GaAs core, $\mathrm{Al}_{0.3} \mathrm{Ga}_{0.7} \mathrm{As}$ shell thick $t_{\text {shell }}=8.6 \mathrm{~nm}$, and GaAs supershell thick $t_{\text {supershell }}=1.7 \mathrm{~nm}$; the overall diameter is then defined as $D=2\left(r+t_{\text {shell }}+t_{\text {supershell }}\right)=151 \mathrm{~nm}$. The NW is $. L=5.19 \mu \mathrm{m}$. long, and it is vertically standing on Si substrate, Fig. 2a. These properties are obtained from the growth parameters and SEM analysis explained in detail in [17]; a SEM image of the NWs that we model is shown in Fig. 1c. Note that during the fabrication, there are also thin AlGaAs and GaAs layers formed on the substrate, that arise from the shell 

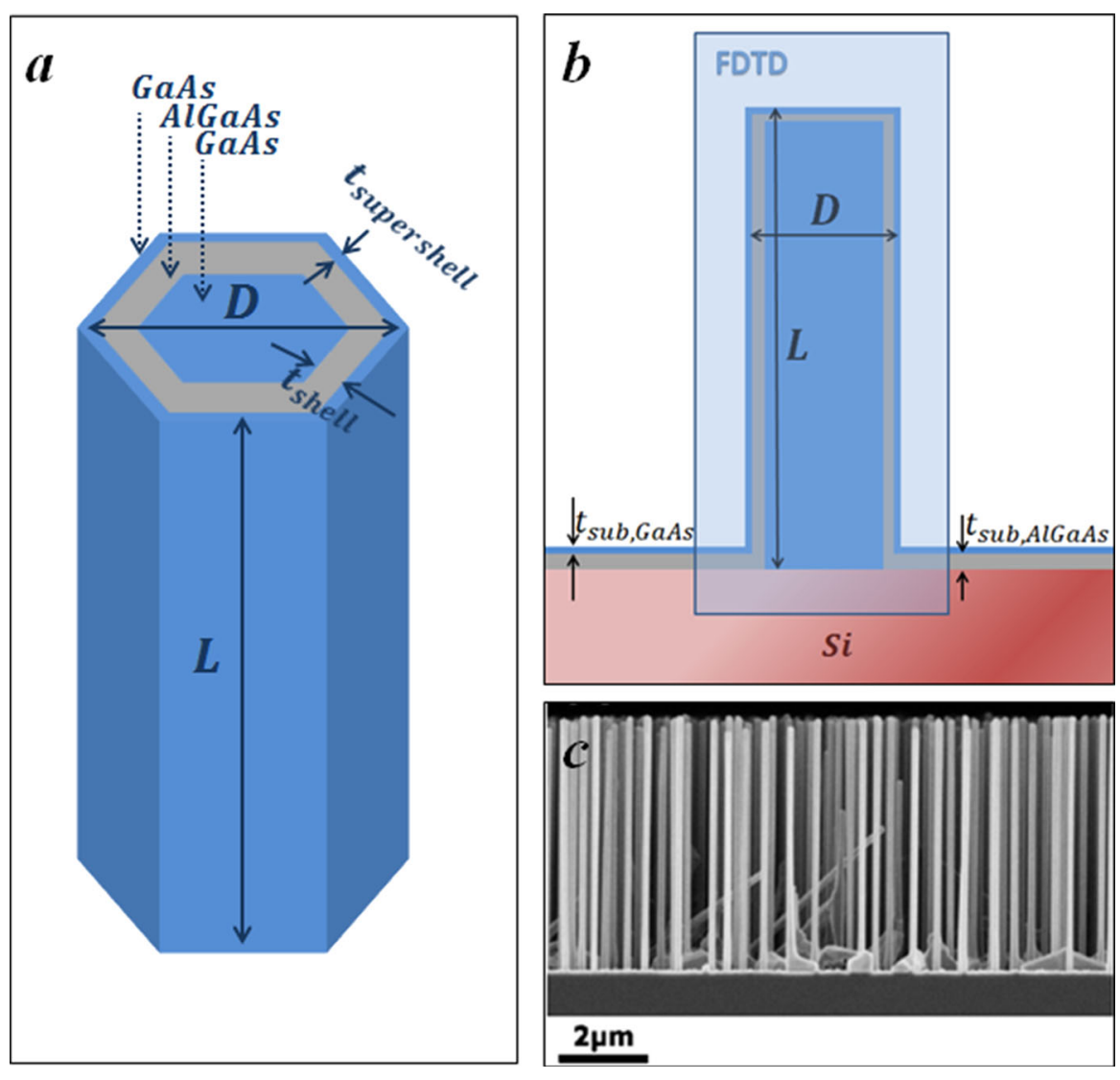

Fig. 1 (a) 3D schematic of the NW investigated: it is defined by GaAs core, the overall diameter $D=$ $151 \mathrm{~nm}$, the length $L=5.19 \mu \mathrm{m}$, the AlGaAs shell $t_{\text {shell }}=8.6 \mathrm{~nm}$, and the GaAs supershell $t_{\text {supershell }}=$ $1.7 \mathrm{~nm}$. (b) $x z$ cross section of the modeled sample, with the FDTD region. (c) SEM image of the sample [17]

and supershell formation, respectively; their dimensions are $t_{s u b, A l G a A s}=38 \mathrm{~nm}$, and $t_{s u b, G a A s}=7.5 \mathrm{~nm}$, and are taken into account in the simulation.

If the light is impinging under normal incidence, the scattering from the end-facets allows for the weakly guided waveguide mode excitation. The energy of these modes is confined on the borders of the NW, thus leading to significant absorption enhancement under the bandgap wavelength. This can be simulated by using a commercial simulator from Lumerical, based on 3D Finite Difference Time Domain (FDTD) method [29]. Firstly, a single NW is surrounded by a simulation region defined by perfectly matched layers (PMLs) in all directions. The special care must be taken to ensure the stability of the simulations. Namely, PMLs are artificial absorbing layers conventionally used in open boundary simulations to truncate the simulation region. Ideally, they should not reflect any electromagnetic field, but due to the computational errors in their proximity, small reflections could lead to errors and instabilities of the calculation. Specifically, if the PMLs are reflecting evanescent fields back into the simulation region, the results 

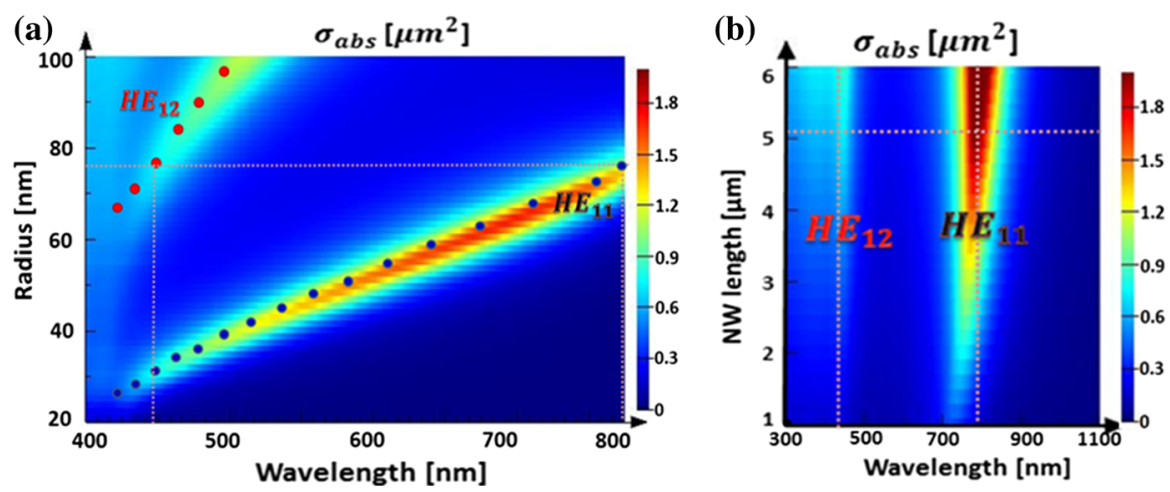

Fig. 2 (a) Absorption cross section spectral dependence on the NW radius defined as $D / 2$; the superposed dots are the dispersion relations in GaAs infinite cylindrical waveguide, which were obtained by analytically solving the Maxwell equations (not shown here); (b) absorption cross section spectral dependence on the NW length $L$. (Color figure online)

will be completely unreliable. Therefore, a special care must be taken to define PML position at least half the maximum wavelength from the surface of the nanostructure. Since the subject here are the visible and near-infrared electromagnetic properties of the NWs, the wavelength range is $300-1100 \mathrm{~nm}$, therefore PMLs are at least $550 \mathrm{~nm}$ distant from the NW borders. In order to discriminate the simulation region with the total field from the one with the scattered field, a total-field scattered source (TFSF) is used to illuminate NW in this spectral range. For a single, stand-alone NW the typical quantity that can provide the mode wavelength excitation and efficiency is the absorption cross section $\sigma_{a b s}$, defined as the ratio of the power absorbed by the NW $P$, and the source intensity $I$; the nanoscale geometries $\sigma_{a b s}$ is usually expressed in $\mu \mathrm{m}^{2}$. $\sigma_{a b s}$ can be calculated by surrounding the NW with a box of monitors in the total-field region, i.e., "inside" the TFSF source; these six monitors calculate the power flow in the box across the spectral range.

In Fig. 2a, we show the simulated dependence of $\sigma_{a b s}$ on the NW radius (defined as $\sim D / 2$ ), by keeping the parameters $t_{\text {supershell }}, t_{\text {shell }}$ and $L$ constant. The analytical approach with Bessel functions was previously applied for the infinite GaAs cylinders, and the first two-mode dependence on the radius is superposed as shown in Fig. 2a (red and blue dots). We see that the enhanced absorption from FDTD simulations perfectly follows the dispersion relation of $H E_{11}$ and $H E_{12}$ modes from the analytical approach, even though this approach does not include the hexagonal cross section, the finiteness and the existence of shell and supershell. Therefore, we can attribute the enhanced absorption to the excitation of the resonant modes in GaAs NW; these modes under bandgap wavelength are lossy, with the electric field confined on the NW borders $[14,15,17]$. For the given $r \approx 75 \mathrm{~nm}$, the resonant absorption of $H E_{11}$ is around $\lambda=800 \mathrm{~nm}$, while $H E_{12}$ is excited around $\lambda=450 \mathrm{~nm}$. The latter leads to weaker absorption and has 2 maxima in the radial direction (in the waveguide theory, a mode $H E_{l m}$ is defined by $l-t h$ order of the Bessel or Hankel function $m$ maxima in the radial direction). Next, the waveguide mode excitation dependence on the NW height is investigated by varying the NW length from 0 to $6 \mu \mathrm{m}$, while keeping the other 
parameters constant. There is a height threshold under which there is no sufficient phase matching to support the waveguide modes; this is expected due to the transition from the disk to the waveguide [18]. Spectral peaks do not shift considerably as $L$. It should be also added that for thicker and longer NW, there is a large spectral gap between the two modes, meaning that these GaAs-based NW show high spectral selectivity. This was underlined before for Si NWs [13] and can be generalized for other semiconductor NWs.

Finally, for the already fabricated sample, one should expect high $H E_{11}$ absorption enhancement at $800 \mathrm{~nm}$, and lower $H E_{12}$ absorption enhancement at $450 \mathrm{~nm}$.

\section{Photo-Acoustic Spectroscopy}

Here, we apply the photo-acoustic spectroscopy (PAS) to directly characterize the expected resonant absorption peaks in the sample. PAS is a photothermal technique where the light absorption leads to the heat generation, thus changing the thermal state of the sample [30, 31]. The modulation of the light by a chopper heats up and cools down the sample; the corresponding pressure changes in PAS cell are an acoustic signal that can be converted into an electrical signal by a close and sensitive microphone, hidden from the scattering by a tunnel. The models of low-noise preamplifier and microphone are Brüel \& Kjær 2639 and 4166, respectively. The system gives a flat frequency response in the $20 \mathrm{~Hz}-200 \mathrm{kHz}$ range. In Fig. 3, our PAS set-up is shown; the light from Xe lamp is directed to the monochromator that allows for $300-1100 \mathrm{~nm}$ range of excitation; the beam diameter is around $300 \mu \mathrm{m}$. The light is then shined on the sample under normal incidence (i.e., for the configuration where we expect $H E_{11}$ and $H E_{12}$ from Sect. 2). The collected signal directly shows the absorption amplitude and phase, regardless of reflected, transmitted, scattered or diffracted light. Moreover, the chopper is close to the light source and more than $50 \mathrm{~cm}$ distant from the PAS cell;

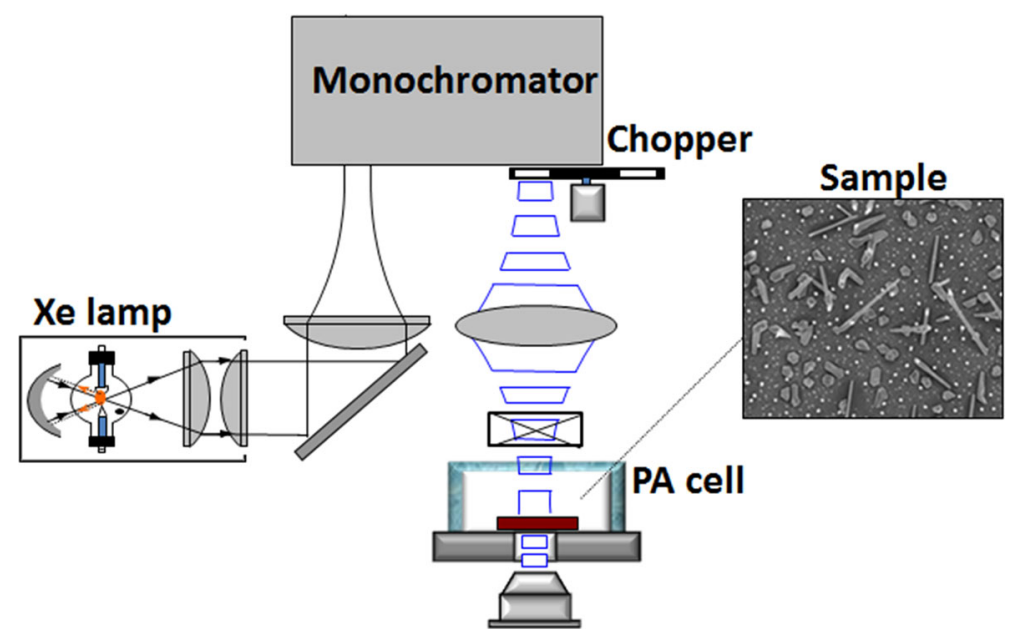

Fig. 3 Photo-acoustic spectroscopy experimental set-up; inset: top SEM image of the sample 
the noise produced by the chopper alone (with the light source switched off) had been previously shown to be negligible, i.e., two orders of magnitude lower than the PAS signal with the light on.

We had previously applied similar PAS set-up to characterize absorption properties of metal-based samples; in [32], we detected surface plasmon polaritons, while in [33-35] we investigated metallic NWs, and helices [36]. Recently, we applied it to measure circular dichroism in similar GaAs-based NW samples that were functionalized by an asymmetrical layer of $\mathrm{Au}$ [21]. This has led us to the idea that also semiconductor samples and their resonant behavior can be characterized by means of the set-up from Fig. 3.

\section{Results and Discussion}

NWs in our sample have insignificant volume compared to the absorbing substrate; this means that the overall absorption will dominantly be flat (non-resonant) over the whole spectral range, if the signal is coming from the whole sample. However, one of the advantages of PAS is that modulation frequency can decide the part of the sample from which the absorption is being collected. This comes from the fact that the thermal diffusion length is inversely proportional to the square root of the modulation frequency [37]. At lower modulation frequencies, thermal signal takes high non-resonant contribution of Si substrate (and thin AlGaAs and GaAs layers), while for high frequencies the thermal diffusion length catches the influence of the NW over its length. In [17], we investigated the PAS spectra dependence on the modulation frequency, and we saw that for the frequencies above $81 \mathrm{~Hz}$ the resonant peaks arise for the similar samples.

In Fig. 4a, we show the PAS signal measured for the NW sample previously investigated. First, Si substrate does not show any resonant feature at neither of frequencies (red lines). For the NW sample at $25 \mathrm{~Hz}$, the resonant contribution is low and the peaks cannot be clearly observed. However, at $225 \mathrm{~Hz}$, both resonant modes appear: $H E_{11}$ at around $800 \mathrm{~nm}$, and $H E_{12}$ at around $450 \mathrm{~nm}$. In Fig. 4b, the simulated absorption
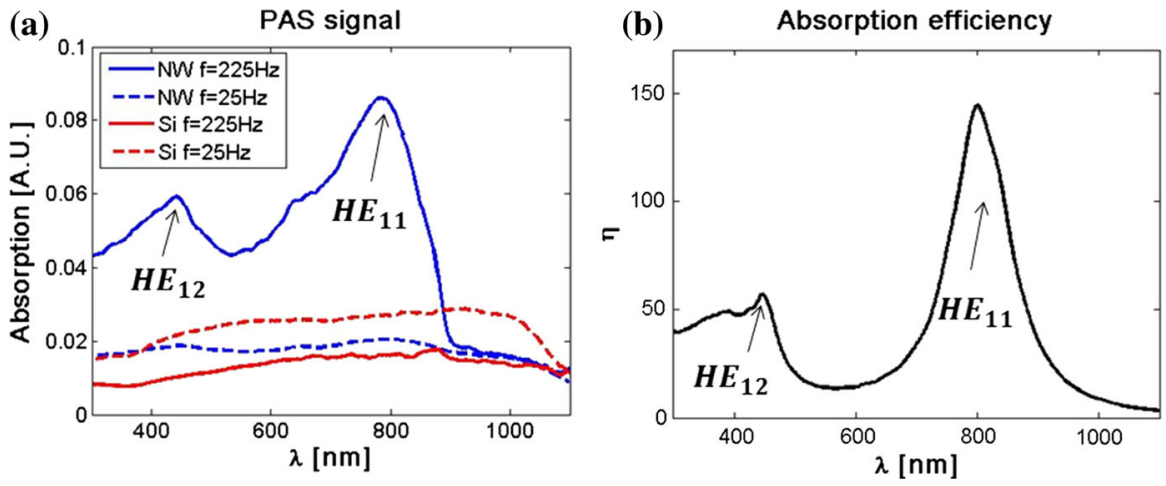

Fig. 4 a PAS signal in arbitrary units at low frequency $(25 \mathrm{~Hz})$ and high frequency $(225 \mathrm{~Hz})$ for the NW sample (blue) and Si substrate (red); b the absorption efficiency of the single NW. (Color figure online) 
efficiency is defined as $\eta=\sigma_{a b s} / \sigma_{\text {cross }}$, where $\sigma_{\text {cross }}$ is geometrical cross section of the single NW. We see that the resonant modes provide high absorption efficiency and that their spectral positions and relative intensities match PAS results very well. However, the experimental peaks are much broader. In [17], we investigated the possible reasons for this broadening: the NW tapering, the fabrication tolerances of the parameters, and the interaction between close NWs. We did similar FDTD simulations of two close NWs, with distance distribution from the top SEM analysis (inset of Fig. 3). These simulations can catch the close NW interaction to some extent and are found to be the main reason for the broadening.

PAS can therefore directly characterize the spectral position and the efficiency of the modes. For a specific application, one can optimize the geometric parameters, e.g., by using methods in [38] or [39]. One of the subjects of ongoing work is the use of the resonant modes for chiral near field formation. Namely, in the points of the confinement, electric and magnetic field possess components that are parallel and out-of-phase. In [19], we shown that the same NW geometry can lead to the near chiral field manipulation by means of the angle of incidence span. Moreover, the NWs can be easily hybridized by thin layers of Au; PAS was used to characterize the absorption spectra of these samples in [21] and the results were in agreement with numerical results from Sect. 2, keeping the same (although broader) waveguide mode peaks. Breaking the symmetry by Au can lead to new perspectives in heat direction manipulation in such samples; the PAS characterization and photo-thermal deflection measurements are subject of future work [40].

\section{Conclusion}

The resonant absorption in GaAs-based NWs fabricated by lithography-free selforganized NW growth was investigated numerically and by means of PAS technique. The results are shown to be in great agreement, meaning that PAS can be used as a non-destructive, low-cost, sensitive and scattering-free method that directly provides the absorption signal. Moreover, there is no complicated post-processing required. Therefore, we firmly believe that PAS can be used as a highly sensitive tool to measure the resonant absorption in nanostructures, for the applications spanning from NW lasers to chirality applications in the samples with broken symmetry.

Funding Academy of Finland Project NESP (294630).

\section{References}

1. L. Cao, J.S. White, J.S. Park, J.A. Schuller, B.M. Clemens, M.L. Brongersma, Engineering light absorption in semiconductor nanowire devices. Nat. Mater. 8, 643-647 (2009)

2. J. Wang, M.S. Gudiksen, X. Duan, Y. Cui, C.M. Lieber, Highly polarized photoluminescence and photodetection from single indium phosphide nanowires. Science 293, 1455-1457 (2001)

3. B. Tian, T.J. Kempa, C.M. Lieber, Single nanowire photovoltaics. Chem. Soc. Rev. 38, 16-24 (2009)

4. O.L. Muskens, J. Gómez Rivas, R.E. Algra, E.P.A.M. Bakkers, A. Lagendijk, Design of light scattering in nanowire materials for photovoltaic applications. Nano Lett. 8, 2638-2642 (2008)

5. J. Wallentin et al., InP nanowire array solar cells achieving $13.8 \%$ efficiency by exceeding the ray optics limit. Science 339, 1057-1060 (2013) 
6. J. Bao, M.A. Zimmler, F. Capasso, X. Wang, Z.F. Ren, Broadband ZnO single-nanowire light-emitting diode. Nano Lett. 6, 1719-17222 (2006)

7. M.H. Huang et al., Room-temperature ultraviolet nanowire nanolasers. Science 292, 1897-1899 (2001)

8. Y. Ahn, J. Dunning, J. Park, Scanning photocurrent imaging and electronic band studies in silicon nanowire field effect transistors. Nano Lett. 5, 1367-1370 (2005)

9. A.B. Greytak, C.J. Barrelet, Y. Li, C.M. Lieber, Semiconductor nanowire laser and nanowire waveguide electro-optic modulators. Appl. Phys. Lett. 87, 151103 (2005)

10. B. Mayer et al., Lasing from individual GaAs-AlGaAs core-shell nanowires up to room temperature. Nat. Commun. 4, 2931 (2013)

11. B. Mayer et al., Monolithically integrated high-beta nanowire lasers on silicon. Nano Lett. 16, 152-156 (2016)

12. I. Åberg et al., A GaAs nanowire array solar cell with $15.3 \%$ efficiency at 1 sun. IEEE J. Photovolt. 6, 185 (2016)

13. B. Wang, P.W. Leu, Tunable and selective resonant absorption in vertical nanowires. Opt. Lett. 37, 3754-3758 (2012)

14. K.T. Fountaine, W.S. Whitney, H.A. Atwater, Resonant absorption in semiconductor nanowires and nanowire arrays: relating leaky waveguide modes to Bloch photonic crystal modes. J. Appl. Phys. 116, $153106(2014)$

15. D.R. Abujetas, R. Paniagua-Domínguez, J.A. Sánchez-Gil, Unraveling the Janus role of Mie resonances and leaky/guided modes in semiconductor nanowire absorption for enhanced light harvesting. ACS Photonics 2, 921-929 (2015)

16. K.T. Fountaine, W. Cheng, C.R. Bukowsky, H.A. Atwater, Near-unity unselective absorption in sparse InP nanowire arrays. ACS Photonics 3, 1826-1832 (2016)

17. G. Leahu et al., Photo-acoustic spectroscopy revealing resonant absorption of self-assembled GaAsbased nanowires. Sci. Rep. 7, 2833 (2017)

18. M.H. Alizadeh, B.M. Reinhard, Emergence of transverse spin in optical modes of semiconductor nanowires. Opt. Express 24, 8471-8479 (2016)

19. E. Petronijevic, M. Centini, A. Belardini, G. Leahu, T. Hakkarainen, C. Sibilia, Chiral near-field manipulation in Au-GaAs hybrid hexagonal nanowires. Opt. Express 25, 14148-14157 (2017)

20. X. Zhao, M.H. Alizadeh, B.M. Reinhard, Generating optical birefringence and chirality in silicon nanowire dimers. ACS Photonics 4, 2265-2273 (2017)

21. G. Leahu et al., Evidence of optical circular dichroism in GaAs-based nanowires partially covered with gold. Adv. Opt. Mater. 2017, 1601063 (2017)

22. N. Vainorius et al., Observation of type-II recombination in single wurtzite/zinc-blende GaAs heterojunction nanowires. Phys. Rev. B 89, 165423 (2014)

23. S. Arab, M. Yao, C. Zhou, P.D. Dapkus, S.B. Cronin, Doping concentration dependence of the photoluminescence spectra of n-type GaAs nanowires. Appl. Phys. Lett. 108, 182106 (2016)

24. S. Thunich et al., Photocurrent and photoconductance properties of a GaAs nanowire. Appl. Phys. Lett. 95, 83111 (2009)

25. S. Zelewski et al., Photoacoustic spectroscopy of absorption edge for GaAsBi/GaAs nanowires grown on Si substrate. Appl. Phys. Lett. 109, 182106 (2016)

26. T.V. Hakkarainen, A. Schramm, J. Mäkelä, P. Laukkanen, M. Guina, Lithography-free oxide patterns as templates for selfcatalyzed growth of highly uniform GaAs nanowires on $\mathrm{Si}(111)$. Nanotechnology 26, 275301 (2015)

27. S. Zelewski et al., Photoacoustic spectroscopy of absorption edge for GaAsBi/GaAs nanowires grown on Si substrate. Appl. Phys. Lett. 109, 182106 (2016)

28. T.V. Hakkarainen, A. Schramm, J. Mäkelä, P. Laukkanen, M. Guina, Lithography-free oxide patterns as templates for selfcatalyzed growth of highly uniform GaAs nanowires on $\mathrm{Si}(111)$. Nanotechnology 26, 275301 (2015)

29. Lumerical Solutions, Inc. http://www.lumerical.com/tcad-products/fdtd/. Accessed 1 Sept 2017

30. T. Inagaki, K. Kagami, E.T. Arakawa, Photoacoustic observation of nonradiative decay of surface plasmons in silver. Phys. Rev. B 24, 3644R (1981)

31. T. Inagaki, K. Kagami, E.T. Arakawa, Photoacoustic study of surface plasmons in metals. Appl. Opt. 21, 949 (1982)

32. E. Petronijevic, G. Leahu, V. Mussi, C. Sibilia, F.A. Bovino, Photoacoustic technique for the characterization of plasmonic properties of 2D periodic arrays of gold nanoholes. AIP Adv. 7, 025210 (2017) 
33. R. Li Voti et al., Photoacoustic characterization of randomly oriented silver nanowire films. Int. J. Thermophys. 36, 1342-1348 (2015)

34. M.C. Larciprete et al., Infrared properties of randomly oriented silver nanowires. J. Appl. Phys. 112, 083503 (2012)

35. A. Belardini et al., Chiral light intrinsically couples to extrinsic/pseudo-chiral metasurfaces made of tilted gold nanowires. Sci. Rep. 6, 31796 (2016)

36. A. Benedetti et al., Precise detection of circular dichroism in a cluster of nano-helices by photoacoustic measurements. Sci. Rep. 7, 5257 (2017)

37. T. Ma, M. Munidasa, A. Mandelis, Photoacoustic frequency-domain depth profilometry of surfacelayer inhomogeneities: application to laser processed steels. J. Appl. Phys. 71, 6029 (1992)

38. R. Li Voti, Optimization of transparent metal structures by genetic algorithm. Rom. Rep. Phys. 64, 446-466 (2012)

39. E. Petronijevic, C. Sibilia, All-optical tuning of EIT-like dielectric metasurfaces by means of chalcogenide phase change materials. Opt. Express 24, 30411-30420 (2016)

40. G. Leahu et al., Study of thermal and optical properties of $\mathrm{SiO} 2 / \mathrm{GaN}$ opals by photothermal deflection technique. Opt. Quant. Electron. 39, 305-310 (2007) 